\title{
Hadronic B decays to open charm at Belle
}

\author{
Rong-Shyang Lu* (Belle collaboration) \\ Department of Physics, National Taiwan University, Taipei, Taiwan, R.O.C.. \\ E-mail: 'rslu@ehep1.phys.ntu.edu.tw'
}

ABStRaCt: Studies of hadronic $B$ decays to charm-related modes at Belle are presented. The results include the first observation of color-suppressed $\bar{B}^{0} \rightarrow D^{0} X^{0}$ decays, a search for $\bar{B}^{0} \rightarrow D_{S}^{-} h^{+}$, an observation of $B^{-} \rightarrow D_{C P}^{0} K^{-}$, and evidence for $\bar{B}^{0} \rightarrow D^{+} D^{*-}$. The results presented here are all preliminary.

\section{Introduction}

Preliminary results on $B$ decays to charm-related modes at Belle are presented. The data sample was collected with the Belle detector [i] integrated luminosity of $21.3 \mathrm{fb}^{-1}$ at the $\Upsilon(4 S)$ resonance, which contains 22.8 million $B \bar{B}$ pairs, and $2.3 \mathrm{fb}^{-1}$ taken $60 \mathrm{MeV}$ below the resonance. In general, the $B$ candidates are identified by two kinematic variables: the beam-constrained mass, $M_{b c}=$ $\sqrt{\left(E_{\text {beam }}^{\mathrm{CM}}\right)^{2}-\left(p_{B}^{\mathrm{CM}}\right)^{2}}$, and the energy difference, $\Delta E=E_{B}^{\mathrm{CM}}-E_{\text {beam }}^{\mathrm{CM}}$, where $E_{B}^{\mathrm{CM}}$ and $p_{B}^{\mathrm{CM}}$ are the CM energy and momentum of the $\bar{B}^{0}$ candidate and $E_{\text {beam }}^{\mathrm{CM}}=\sqrt{s} / 2=5.29 \mathrm{GeV}$.

\section{First observation of color-suppressed $\bar{B}^{0} \rightarrow D^{0} X^{0}$ decays.}

The decay modes $\bar{B}^{0} \rightarrow D^{(*) 0} X^{0}$, where $X^{0}$ is a light neutral meson, proceed via an internal spectator diagram, and are expected to be suppressed relative to the external diagram, since the color of the $\bar{u}$ antiquark produced by the weak current must cancel the color of the $c$ quark, as shown in Fig. $i_{i}^{i}$ Studies of such color-suppressed decay modes can be used to test models of hadronic $B$ meson decays and to provide information on final-state interactions. Results

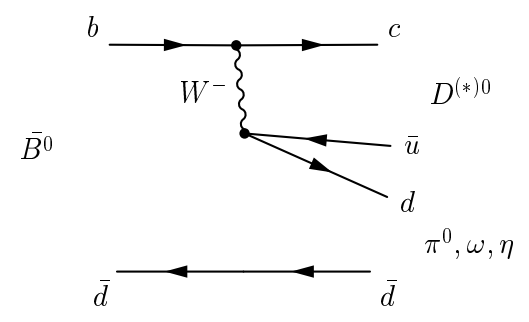

Figure 1: The Feynman diagram for $\bar{B}^{0} \rightarrow D^{(*) 0} X^{0}$ decays. of searches for color-suppressed $\bar{B}^{0} \rightarrow D^{(*) 0} X^{0}$ decays have been published by the CLEO collaboration [B]i]; however, only upper limits were obtained.

\footnotetext{
${ }^{*}$ Speaker.
} 
In addition to the $q \bar{q}$ background, we observe large background contributions from colorfavored $B \rightarrow D^{(*)}(n \pi)^{-}$decays and cross-talk from $D^{* 0} X^{0}$ to $D^{0} X^{0}$ modes. The $D^{*+} \rho^{-}$mode has the same final state as $D^{0} \omega$ and $D^{0} \eta$, but can be suppressed by $\omega$ or $\eta$ mass window cuts. The $D^{(*) 0} \rho^{-}$final state contaminates the $D^{(*) 0} \pi^{0}$ and $D^{(*) 0} \eta$ modes if the $\rho^{-}$decays to a fast $\pi^{0}$. About half of these events are removed by explicitly reconstructing the $D^{(*) 0} \rho^{-}$final state. The contributions of these backgrounds in the $\eta$ channel, as well as the feed-across from the $D^{(*) 0} \pi^{0}$ mode, is minimized by a $\pi^{0}$ veto. We also check for background contributions from $\bar{B} \rightarrow D^{(*) 0} \rho^{--}\left(\rho^{\prime-} \rightarrow \omega \pi^{-}\right)$decays, which have recently been observed by CLEO [4]. This twobody decay produces high-momentum $D^{(*) 0} \mathrm{~s}$ and $\omega$ s that can fake signal events. MonteCarlo studies indicate that the remaining background events can be distinguished from signal events by fitting the $\Delta E$ distribution.

The $\Delta E$ distributions for the various $D^{(*) 0} X^{0}$ decays are shown in Fig. 2 . Table $\left.\right|_{1} ^{1}$ summarizes the results for each $D^{(*) 0} X^{0}$ mode. In general, the branching fractions are higher than theory

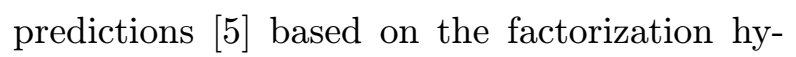
pothesis. This may be accounted for by additional corrections to the factorization models, or by non-factorizable effects, such as final state interactions.

\begin{tabular}{llcclc} 
Mode & Signal Yield & Significance & Efficiency $(\%)$ & $\mathcal{B}\left(\times 10^{-4}\right)$ & UL $\left(\times 10^{-4}\right)$ \\
\hline $\bar{B}^{0} \rightarrow D^{0} \pi^{0}$ & $127.6_{-17.9}^{+18.5+11.6}$ & 7.9 & 1.93 & $2.9_{-0.3}^{+0.4} \pm 0.6$ & - \\
$\bar{B}^{0} \rightarrow D^{* 0} \pi^{0}$ & $17.1_{-5.9}^{+6.6}+.6 .6$ & 3.2 & 0.49 & $1.5_{-0.5}^{+0.6}+0.3$ & 2.3 \\
\hline $\bar{B}^{0} \rightarrow D^{0} \eta$ & $25.7_{-7.4}^{+8.4}+{ }_{-2.8}^{+3.0}$ & 3.8 & 0.79 & $1.4_{-0.4}^{+0.5} \pm 0.2$ & 2.1 \\
$\bar{B}^{0} \rightarrow D^{* 0} \eta$ & $7.7_{-2.7}^{+3.4}{ }_{-0.8}^{+0.7}$ & 3.6 & 0.22 & $1.5_{-0.6}^{+0.7} \pm 0.4$ & 2.7 \\
\hline $\bar{B}^{0} \rightarrow D^{0} \omega$ & $30.2_{-7.8}^{+8.6+3.1}$ & 4.7 & 0.80 & $1.7_{-0.4}^{+0.5}+0.3$ & - \\
$\bar{B}^{0} \rightarrow D^{* 0} \omega$ & $17.7_{-5.8}^{+6.5}+2.3$ & 4.3 & 0.23 & $3.4_{-1.1}^{+1.3} \pm 0.8$ & -
\end{tabular}

Table 1: The obtained signal yield, statistical significance, efficiency including the sub-decay branching fractions, branching fraction $(\mathcal{B})$, and $90 \%$ confidence level upper limit (UL) for each $\bar{B}^{0} \rightarrow D^{(*) 0} X^{0}$ decay mode.
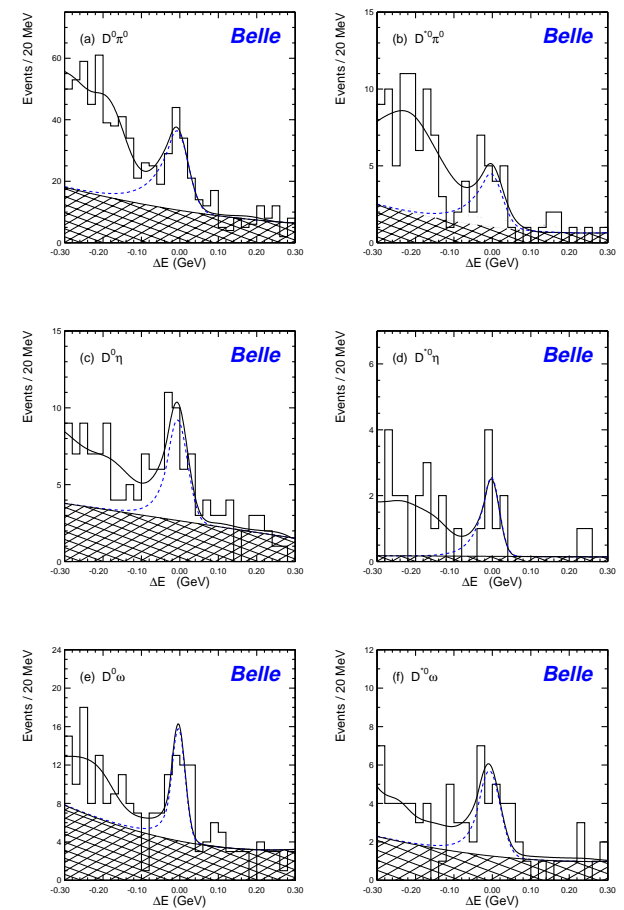

Figure 2: The $\Delta E$ distribution for (a) $D^{0} \pi^{0}$, (b) $D^{* 0} \pi^{0}$, (c) $D^{0} \eta$, (d) $D^{* 0} \eta$, (e) $D^{0} \omega$, and (f) $D^{* 0} \omega$. The solid line shows the fitting result. The dashed line shows the sum of the signal component and the combinatorial background component. The combinatorial component is shown separately as the crosshatched area. 


\section{Search of $\bar{B}^{0} \rightarrow D_{s}^{-} h^{+}$decays.}

The $\bar{B}^{0} \rightarrow D_{s}^{-} \pi^{+}$decay proceeds via the $b \rightarrow$ $u$ transition. Therefore, a measurement of the branching fraction, $\mathcal{B}\left(\bar{B}^{0} \rightarrow D_{s}^{-} \pi^{+}\right)$, gives access to the CKM matrix element, $\left|V_{u b}\right|[\overline{6} \bar{i} \mid$. One advantage of measuring $V_{u b}$ from the $B^{0} \rightarrow$ $D_{s}^{+} \pi^{-}$rate is that a tree-level diagram dominates the process, and there is no contribution from the penguin diagram. The $\bar{B}^{0} \rightarrow D_{s}^{+} K^{-}$ decay proceeds via $W$ exchange, and is ex-

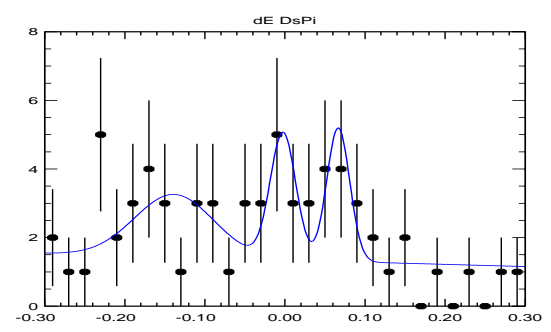

Figure 3: The $\Delta E$ distribution for $\bar{B}^{0} \rightarrow$ $D_{s}^{-} \pi^{+}$search.

pected to be highly suppressed, by a factor of $10^{-4}$, compared to the tree diagram. Therefore, searches for $\bar{B}^{0} \rightarrow D_{s}^{+} K^{-}$decays provide a probe of the final state interactions, which may enhance the branching fraction by several orders of magnitude.

The $D_{s}^{-}$candidates are reconstructed in the $D_{s}^{-} \rightarrow \phi \pi^{-}, K_{S}^{0} K^{-}$, and $K^{* 0} K^{-}$decay modes. We combine $D_{s}^{-}$and $h^{+}$mesons to form $\bar{B}$ candidates, where $h^{+}$is either $\pi^{+}$or $K^{+}$. Fig. ' ${ }_{-1}^{\overline{3}}$ ' shows the $\Delta E$ distribution for $\bar{B}^{0} \rightarrow D_{s}^{-} \pi^{+}$candidates. The three peaks, from left to right, correspond to the $B^{0} \rightarrow D^{-} \rho^{+}$background, $D_{s}^{-} \pi^{+}$signal, and $B^{0} \rightarrow D^{-} \pi^{+}$ background peak. The results of $\bar{B}^{0} \rightarrow D_{s}^{-} h^{+}$search are summarized in Table ${ }_{-1}^{1}$ with $90 \%$ C.L. upper limits. We obtained an improved upper limit on $\bar{B}^{0} \rightarrow D_{s}^{+} K^{-}$and a limit of $\left|V_{u b}\right|<0.0065$, which is consistent with the PDG $\left[\overline{q_{1}}\right]$ value, $\left|V_{u b}\right|=0.005$.

\begin{tabular}{lcccc} 
Mode & Signal Yield & Upper limit & CLEO UL & Theory pred. \\
\hline $\bar{B}^{0} \rightarrow D_{s}^{-} \pi^{+}$ & $7.3 \pm 3.7$ & $<16 \times 10^{-5}$ & $7.5 \times 10^{-5 \dagger}$ & $4.5 \times 10^{-5 *}$ \\
$\bar{B}^{0} \rightarrow D_{s}^{-} K^{+}$ & $2.2 \pm 2.5$ & $<14 \times 10^{-5}$ & $24 \times 10^{-5 \ddagger}$ & $0.66 \times 10^{-5 \S}$ \\
\hline${ }^{\dagger} @$ APS, 2000; ${ }^{\ddagger}$ PRL B319, 1993; ${ }^{*}$ T.Kurimoto @BCP4 ${ }^{\S}$ PLB, B406 1997
\end{tabular}

Table 2: Summary of the $\bar{B}^{0} \rightarrow D_{s}^{-} h^{+}$search results.

\section{Observation of $B^{-} \rightarrow D_{C P}^{0} K^{-}$decays.}

The extraction of the $\phi_{3}$ angle in the CKM

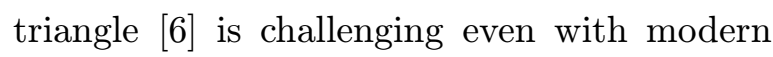
high-luminosity $B$ factories. Recent theoretical work has demonstrated that $\phi_{3}$ can be determined from $B^{-} \rightarrow D K^{-}$decays by using the interference between the $b \rightarrow c$ and $b \rightarrow u$ processes, which are shown in Fig. 花. The an-

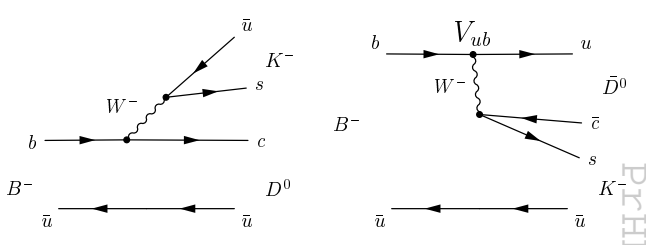

Figure 4: $B^{-} \rightarrow D K^{-}$decay amplitudes. gle $\phi_{3}$ can be extracted from $B^{-} \rightarrow D_{1,2} K^{-}$decays, where $D_{1}$ and $D_{2}$ are $\mathrm{CP}=+$ and - eigenstates, respectively. Assuming the absence of $D^{0}-\bar{D}^{0}$ mixing, the observables sensitive to $\mathrm{CP}$ violation that are used to extract the angle $\phi_{3}$ are :

$$
\mathcal{A}_{1,2} \equiv \frac{\mathcal{B}\left(B^{-} \rightarrow D_{1,2} K^{-}\right)-\mathcal{B}\left(B^{+} \rightarrow D_{1,2} K^{+}\right)}{\mathcal{B}\left(B^{-} \rightarrow D_{1,2} K^{-}\right)+\mathcal{B}\left(B^{+} \rightarrow D_{1,2} K^{+}\right)}=\frac{2 r \sin \delta^{\prime} \sin \phi_{3}}{1+r^{2}+2 r \cos \delta^{\prime} \cos \phi_{3}}
$$




$$
\begin{aligned}
R_{1,2} & \equiv \frac{\frac{\mathcal{B}\left(B^{-} \rightarrow D_{1,2} K^{-}\right)+\mathcal{B}\left(B^{+} \rightarrow D_{1,2} K^{+}\right)}{\mathcal{B}\left(B^{-} \rightarrow D_{1,2} \pi^{-}\right)+\mathcal{B}\left(B^{+} \rightarrow D_{1,2} \pi^{+}\right)}}{\frac{\mathcal{B}\left(B^{-} \rightarrow D^{0} K^{-}\right)+\mathcal{B}\left(B^{+} \rightarrow \bar{D}^{0} K^{+}\right)}{\mathcal{B}\left(B^{-} \rightarrow D^{0} \pi^{-}\right)+\mathcal{B}\left(B^{+} \rightarrow \bar{D}^{0} \pi^{+}\right)}}=1+r^{2}+2 r \cos \delta^{\prime} \cos \phi_{3} \\
\delta^{\prime} & =\delta \text { for } D_{1} ; \quad \delta+\pi \text { for } D_{2} .
\end{aligned}
$$

Here $r$ denotes the ratio of the amplitudes, $r \equiv A\left(B^{-} \rightarrow \bar{D}^{0} K^{-}\right) / A\left(B^{-} \rightarrow D^{0} K^{-}\right)$, and $\delta$ is the strong phase difference. Here, we report on a study of $B^{-} \rightarrow D K^{-}$decays, where the $D^{0}$ meson decays into a flavor-specific state, $K^{-} \pi^{+}$, or into a $\mathrm{CP}=+1$ eigenstate, $K^{-} K^{+}$or $\pi^{-} \pi^{+}$.
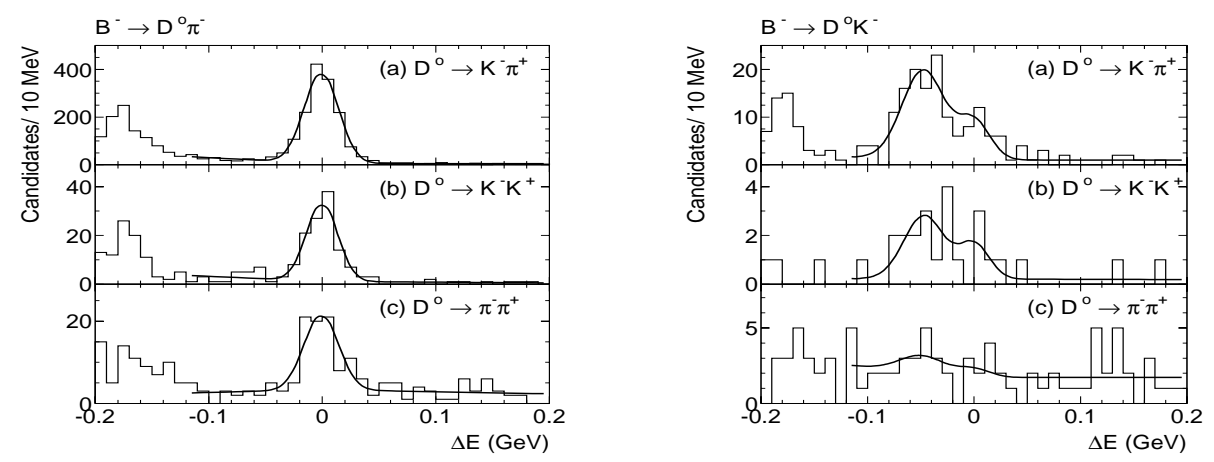

Figure 5: The $\Delta E$ distribution for $B^{-} \rightarrow D^{0} \pi^{-}$and $B^{-} \rightarrow D^{0} K^{-}$candidates.

In order to identify $B^{-} \rightarrow D K^{-}\left(\pi^{-}\right)$samples, we looked at the $\Delta E$ distributions shown in Fig. 15.5 for various modes. With the pion mass assumption, $B^{-} \rightarrow D \pi^{-}$events peak at $\Delta E=0$, while the $D K^{-}$peak is shifted to $\Delta E=-49 \mathrm{MeV}$. The signal yields are $12.3 \pm 3.9$ events with a significance of 4.3 for $B^{-} \rightarrow D^{0}\left(K^{+} K^{-}\right) K^{-}$and $4.9 \pm 5.4$ events with a significance of 0.94 for the $B^{-} \rightarrow D^{0}\left(\pi^{+} \pi^{-}\right) K^{-}$mode.

Using the signal yields in the $D_{C P} K$ modes, the $90 \%$ C.L. range for the asymmetry is determined to be $-0.78<\mathcal{A}_{1}<0.94$. The double ratio $\left(R_{1}\right)$ is obtained to be $R_{1}=$ $1.39 \pm 0.53$ (stat. $) \pm 0.26$ (syst.). The results for $R_{1}$ and $\mathcal{A}_{1}$ are in good agreement with the Standard Model prediction.

\section{Evidence of $\bar{B}^{0} \rightarrow D^{+} D^{*-}$ decays.}

The decays $B^{0} \rightarrow D^{ \pm} D^{* \mp}$ are of special interest for the measurement of the CP violation parameter $\sin 2 \phi_{1}$. A full reconstruction of both charm mesons results a small overall efficiency for $B$ reconstruction. In this analysis the $D^{+}$is fully reconstructed, while only the slow pion from the $D^{*-}$ decay is used. The slow pion, $\pi_{\text {slow }}^{-}$, from the $D^{*-}$ decay approximately retains the $D^{*-}$ momentum direction because of the small energy release in the decay and the polarization of the $D^{*-}$ meson. Thus, the angle $\alpha$ between the slow pion and the $D^{+}$meson can be employed as a signature of the studied decay.

The continuum contribution is subtracted using data taken at energies below the threshold of $B \bar{B}$ production. Data are further divided into two data sets, with and without 
a high-momentum lepton tag. The high-momentum lepton tag suppresses the continuum background to a negligible level, and can be used as a tagging particle for future CP studies.

Fig. $\overline{6}_{1}^{1}$ shows the $\cos \alpha$ distribution for the data with and without a lepton tag; the signal yields are $244 \pm 87$ and $35.8 \pm 11.3$ events, respectively. The systematic error is found to be dominated by the treatment of background. The peaking backgrounds from $B^{0} \rightarrow D^{*+} D^{*-}$ and $D_{s}^{(*)+} D^{*-}$ decays are estimated by Monte-Carlo simulation and subtracted from the data. Other possible backgrounds from $B$ decays to doublecharm mesons, like $D_{s 1}^{+} D^{(*)-}, D_{1}(2420) D+$, and $D_{2}^{*}(2460) D^{(*)+}$, are estimated to be negiligible assuming $10^{-3}$ branching ratios for each mode. The possible contributions of these modes are also included in the systematic error. The combined results from two data samples give the branching fraction,

$$
\mathcal{B}\left(\bar{B}^{0} \rightarrow D^{+} D^{*-}\right)=\left(0.92 \pm 0.23_{-0.30}^{+0.34}\right) \times 10^{-3} .
$$

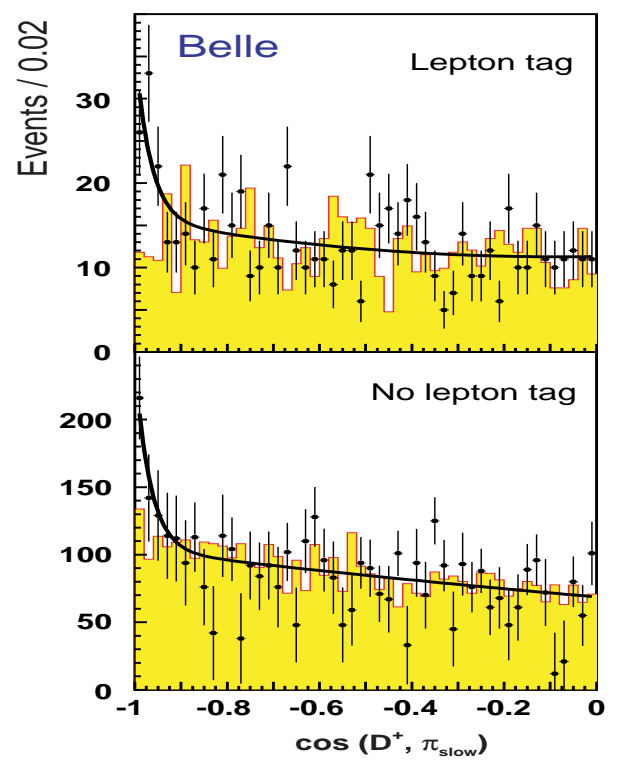

Figure 6: The $\cos \alpha$ distribution for the data set (a) with a lepton and (b) without a high-momentum lepton.

\section{Conclusion}

We have presented new Belle results for $B$ decays with charm mesons. The color-suppressed $\bar{B}^{0} \rightarrow D^{0} \pi^{0}, D^{0} \omega$, and $D^{* 0} \omega$ modes were observed for the first time. We also obtained an improved upper limit on the $\bar{B}^{0} \rightarrow D_{s}^{+} K^{-}$mode. A study of the extraction of the CKM angle $\phi_{3}$ has been presented using the $B^{-} \rightarrow D_{C P}^{0} K^{-}$decay; we observed for the first time $\bar{B}^{0} \rightarrow D_{C P}^{0} K^{-}$where $D^{0} \rightarrow K^{+} K^{-}$. Evidence for $\bar{B}^{0} \rightarrow D^{+} D^{*-}$ has been seen with a partial reconstruction method; this mode can be used in future $\mathrm{CP}$ violation studies.

\section{References}

[1] Belle Collaboration, A. Abashian et al., KEK Progress Report 2000-4 (2000), to be published in Nucl. Inst. and Meth. A.

[2] KEKB B Factory Design Report, KEK Report 95-7 (1995), unpublished; Y. Funakoshi et al., Proc. 2000 European Particle Accelerator Conference, Vienna (2000).

[3] CLEO Collaboration, T. Bergfeld, et al., Phys. Rev. D 57, 5363 (1998).

[4] CLEO Collaboration, J. Alexander, et al., hep-ex/0103021, submitted to Phys. Rev. D.

[5] M. Neubert and B. Stech, hep-ph/9705292, in Heavy Flavours, edited by A. J. Buras and M. Lindner, 2nd ed. (World Scientific, Singapore).

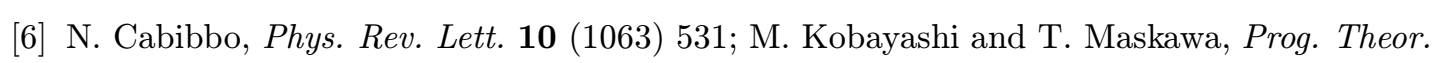
- - -

[7] Particle Data Group, D.E. Groom et al., Eur. Phys. J. C15, 1 (2000). 